\title{
SUPPLY CHAIN MANAGEMENT AND DISTRIBUTED MANUFACTURING IN THE AUTOMOTIVE INDUSTRY
}

\author{
Gerhard Schaub, Erich Krauß
}

Diconsult GmbH, Grubmühlerfeldstr. 14c,82131 Gauting, GERMANY, GS@diconsult.de

DaimlerChrysler AG, 68299 Mannheim, GERMANY, Erich.krauss@daimlerchrysler.com

\begin{abstract}
There is a novel approach for realising distributed manufacturing systems based on an object-oriented database which entirely runs in the random access memories (RAM) of the involved distributed computers. This is a very advantageous way to reduce costs for the design, implementation, operation and maintenance of a Supply Chain Management system. The example of an automotive parts' supplier shows the capability and benefits of the new concept especially if the manufacturing is performed by different companies in different countries. The object-oriented database fits seamlessly into the Internet/Intranet approach and provides chances to realise virtual enterprises with minimum efforts and expenditures. Hence, this system is not only interesting for big companies but also for small and medium.
\end{abstract}

\section{INTRODUCTION}

Car manufacturers are very innovative and try to find economical solutions wherever possible. Hence, they are often the trailblazers for novel technology. In the Supply Chain Management area DaimlerChrysler pursued a new path to realise a "distributed information management system" by using an object-oriented real-time database (OO-RT-DB) which entirely runs in the main memory. In spite of that it is more reliable, faster and cheaper than a relational database management system (RDBMS). However, the main advantage is that a large scaled information system is cut into many pieces which are easy to handle in contrast to a traditional centralised database system. This will be shown on a distributed wire harness production for the new truck generation ACTROS and ATEGO.

To avoid expensive warehouses DaimlerChrysler uses Just-in-Time (JIT) production which means the sub-suppliers have to deliver the right components at the right time directly to the assembly line. The latest trend goes towards customer-tailored-cars which means that a car is no longer a mass-produced article but assembled by components that are produced in pieces of one. On top of this the production partially is given to low-wages countries and at the same time the delivery periods are tried to be decreased noticeable. Hence, to reach all these goals together a powerful logistics and IT-system was necessary which was built by Diconsult $\mathrm{GmbH}$. 
The new trucks are assembled in Wörth (Germany) and the wire harnesses are produced $30 \mathrm{~km}$ away in Mannheim (Germany) and parts of it in Liberec (Czech Republic) and other places (see Figure 1). But there is a must to be able to produce complete components near the assembly line to deliver in three hours wire harnesses that has been damaged during assembly or shortly before. Hence, there have to be production sites which are specialised in manufacturing modules and variants but the site in Mannheim must be able to do everything. This is also an advantage for distributing and balancing the workload. The entire system is controlled by DaimlerChrysler in Mannheim.

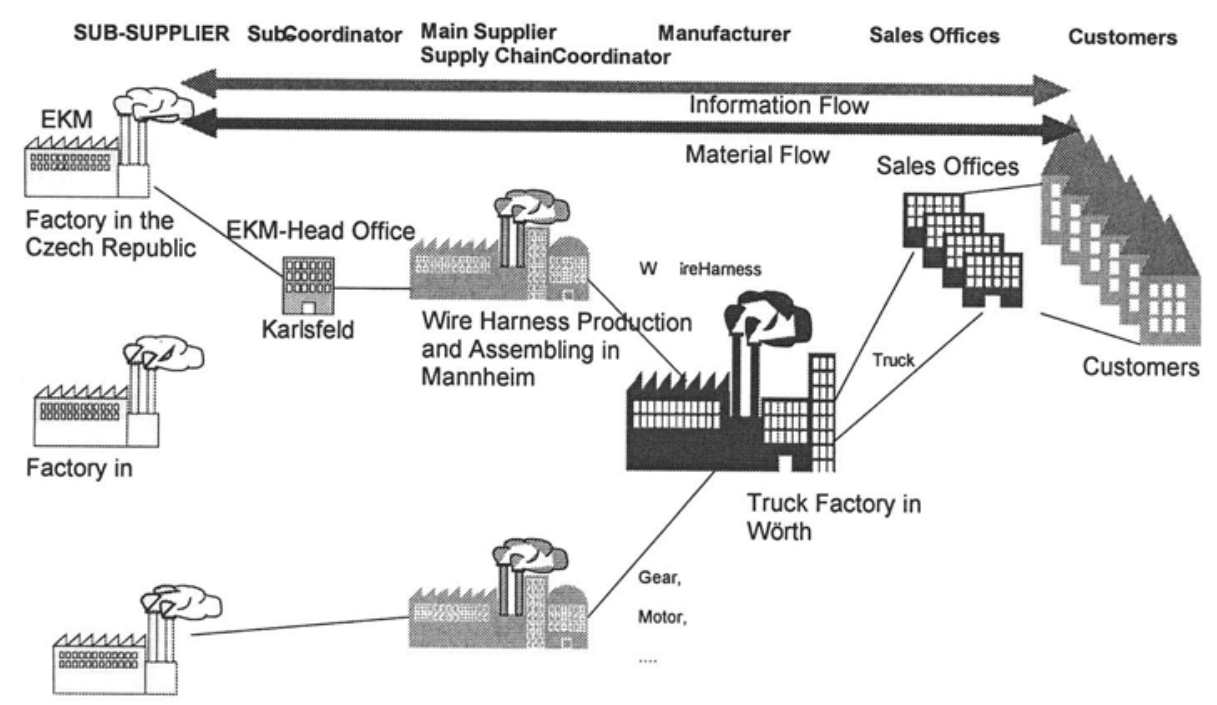

Figure 1 - Supply Chain for Wire Harness Production

\section{The Virtual Enterprise for wire harness production}

The high demands are firstly to deliver the customised wire harnesses just-in-time to the assembly lines of the truck manufacturer, secondly to have only some days to produce it, thirdly the extensive manual work and the strong competition needs an additional production in low-wage countries and fourthly the high variety of variants. The repeatability is so little that each wire harness is not manufactured before the order is received from the truck manufacturer. That means a total production-ondemand is needed.

Every working day more than 2,000 variants are produced ( 9 for one truck) consisting of 78,000 modules built with 740,000 single work steps. To keep the whole system controlled more than 6,000 EDI calls are transmitted daily from the truck factory to the supplier. The supplier himself has to control all the sub-suppliers.

The entire production system in Mannheim, in Karlsfeld, and in Liberec was built by using the object-oriented real-time database running on PC's. 


\section{OBJECT-ORIENTED REAL-TIME DATABASE (OO-RT-DB)}

\section{The Object-Oriented Data Server}

In many applications the RDBMS are suitable, but not so in distributed manufacturing systems. There are too many "equi joins" necessary because the customer orders are related to production orders, work plans, bill-of-materials, and other tables depending on the project. Not seldom the applied ERP or MES systems need to much time to process all data.

In contrast to this the OO-RT-DB is not only more than 30 times faster but also allows to cut large scaled systems into easy to handle pieces which are named OOdata servers. They are all based on the same program code which is able to satisfy the following requirements:

- to manage the data (create, delete, change, copy data) in accordance to the orders sent by the clients or agents

- to co-ordinate all the requests occurring parallel from more than one client; In contrast to a RDBMS the server is not blocked during a message sequence with one client. The requests can come from local clients or remote clients via network and TCP/IP sockets communication

- automatically start/shutdown together with the computer start and shutdown

- to save all data intelligently on a hard disk drive, not to loose any data even if the power supply breaks down or other disturbances occur. In the end, the OORT-DB has advantages over a RTBMS in this respect as well.

The server gets specific properties, such as data structure, hierarchical structure, sort criteria, keys, etc., via a parameterisation of the appertaining tables, for instance if the server is an order or an warehouse server etc. This creation of a new server is made very easy by means of a graphical user interface.

All these server objects (e.g. order, BOM, article basic data, ...) can be separately designed, developed, tested and maintained. That is very important and a tremendous advantage when establishing very fast good working software.

\section{The Object-Oriented Control of the System}

The most important and most difficult part of a project is to cut a project into pieces which are independent objects. For this purpose one has to understand the current workflow and has to change it in accordance with these requirements:

- The pieces of the workflow as a result of the new approach must be straight forward and without back loops

- They must be designed so that they are independent of each other

- They are always individually designed together with the company and people affected by the new workflow

- They must be simple so that the employees are able to follow them and the easier the workflow the better the whole project works at the end

Generally speaking there are always clients, agents, data servers and actions of persons occurring in a workflow. 
The clients are graphical user interfaces together with algorithms or other systems, for example shop floor terminals which request data from the servers to process them and to give the order to the server to store them again (read, change, write data). The client is also a program which takes the initiative and which can control some parts of the system. A client only speaks to a server.

The servers only fulfil the requests of the clients and the agents. There is no inherent initiative or control. A server is never transmitting data to another server.

The agents are programs which help in situations where servers want to speak to servers. For instance when a server has to store its data on a server on a back-up computer then an agent reads the data from the original server and stores it on the other one. This is much more secure and cheaper than to use a RAID 5 system.

An agent also plays a crucial role when a legacy system has to be connected. For instance, the agent looks periodically at a file in which the PPC writes all the new data like orders, basic articles, BOM, etc. If data are available the agent converts the data into the right object-oriented structure of the different servers and invokes the server to store that data.

Such a legacy-agent is an appropriate tool to connect all legacy systems in the course of one or two days of programming and testing independent of the data structure of the legacy system.

An agent is also an element which controls the system. The agent asks for data from a certain server (e.g. order server) looking for example whether there are orders with a certain status. The agent sends a certain query which is answered by the database server.

An agent is also able to receive events and to react in a certain and appropriate way. But to control a system through events should be avoided whenever it is possible because this is the way to get an old fashioned spaghetti like system.

It is almost always possible to decouple a system through cyclic polling. This sounds strange but in reality it is an excellent method to cut a difficult system into autonomous subsystems that are easy to handle (see Figure 2). It avoids a lot of problems since a system designed that way can never be overloaded and crash because of too many events being received.

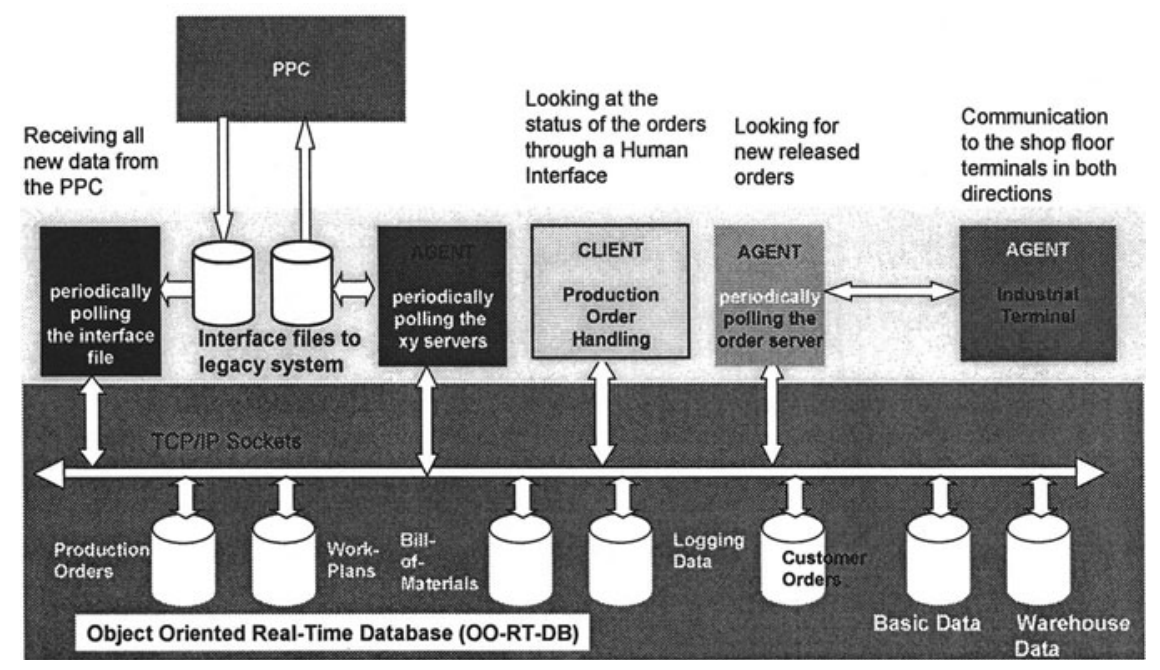

Figure 2 - Object-Oriented (Software) Servers, Clients, and Agents 
All the servers, clients, and agents can run together on one computer but they can also be arbitrarily distributed over a network (LAN, WAN, Internet, etc.) without any additional programming effort because the communication is based on TCP/IP and sockets.

\section{Object-Oriented Communication}

According to the distributed objects we developed a communication tool which is able to deal with all these requirements in an appropriate way.

It has similar objectives as CORBA and DCOM but it easier and faster than either of them. During the ESPRIT project DELPHI we extended the interface so that the OO-RT-DB servers now also have a DCOM and an ODBC Interface (Figure 3).

Beside these two we have developed an Internet interface so that the access to the object-oriented database servers is possible through 4 different interfaces:

- a proprietary interface which is very fast and reliable based on TCP/IP and sockets

- DCOM which is based on the Microsoft technology but now also available for LINUX, HP-UX, IBM-AIX, etc.

- ODBC

- Internet interface to access data of the object-oriented real-time database servers by means of www-browsers.
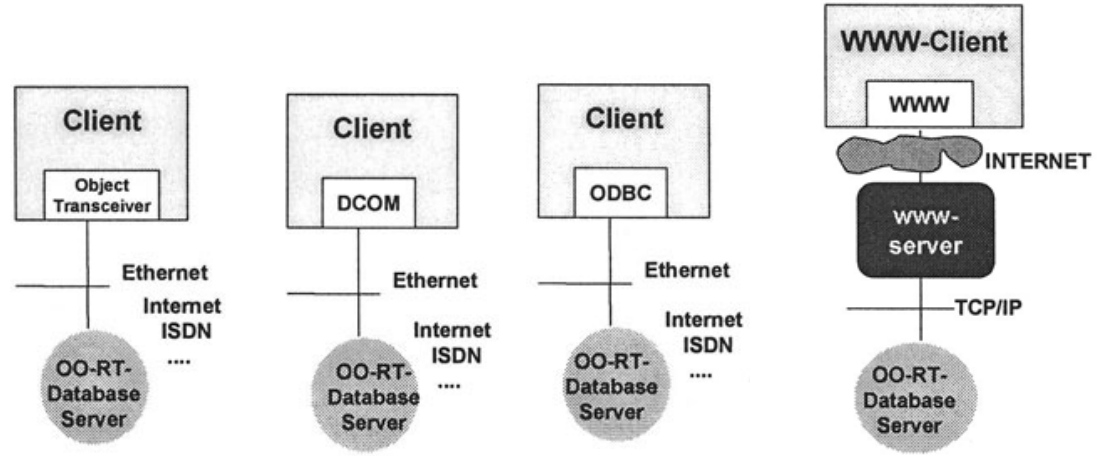

Figure 3- Different ways to communicate within a distributed system

Figure 4 illustrates the hierarchical structure of a truck order that is divided in 9 variant orders which are containing module orders and at least components. All relations are very important to know because the modules for this truck for example have to be produced in different countries and they all have to be assembled in Mannheim at a certain point in time. This moment is not fixed from the beginning of getting the truck order (10 days before delivery) because all orders are daily updated in accordance with the real situation at the truck plant in Wörth. The last update arrives 3 hours before delivery.

Such hierarchical structures are very easy to implement applying the OO-RT-DB and they are very, very fast. This is very necessary because of the 60,000 order updates per day which have to be processed by the system. 


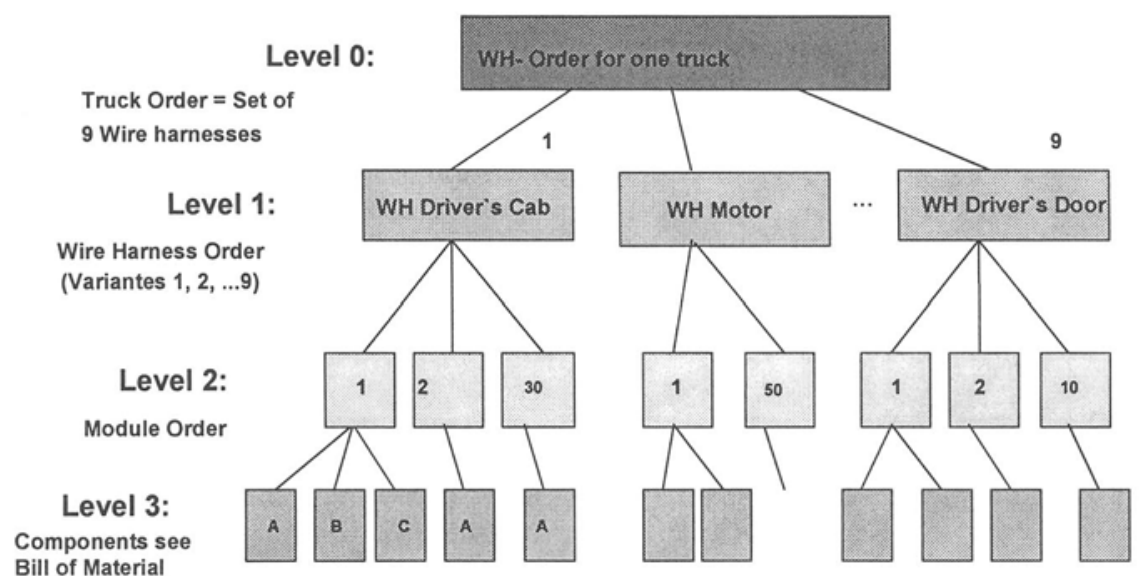

Figure 4 - Hierarchical Structure of an Truck Order

\section{Reliability}

The reliability of an IT-system is the most important feature. As our OO-RT-DB server holds the data in main memory (RAM) for reasons of speed, we had to take special measures to avoid problems in case of a power failure.

Therefore the server automatically writes checkpoints to the hard-disk-drive (HDD) every $\mathbf{x}$ minutes where $\mathrm{x}$ is an adjustable parameter, for example 30 . To shorten the time to write the RAM information to the disk the server information is first copied to another main memory server and from this the data are compressed and written on the HDD. So the original server is only blocked for the time making a main memory copy to another area. This process needs only seconds depending on the amount of data which have to be copied, for example 20 Mbyte need about 2 seconds on a standard PC.

So every 30 minutes only 2 seconds are lost through a checkpoint. The amount of information held in 20 MByte is huge and not to compare with the needed resources of a RDBMS database because there is nearly no overhead. For example, a RDBMS needs 200 times more HDD space and 50 times more RAM.

The average seize of our OO-servers in this project is 10 to 20 MByte and only for huge amount of data it is $100 \mathrm{MByte}$. There are about 50 OO-servers in operation.

Our standard OO-RT-DB server has the following features: All x minutes a checkpoint is written on the hard disk drive (HDD). Between two checkpoints all changes made by clients or agents are logged in a logging file. In the case of a power failure (for example when a computer is switched off by mistake) all the OO-RT-DB servers automatically restore their data by taking the last checkpoint and driving along the logging files so that all inserts, changes, etc. are again taken into account and the previous state is restored exactly as it was before the power breakdown (see Figure 5). There are more specific tricks which are too detailed for this report. 


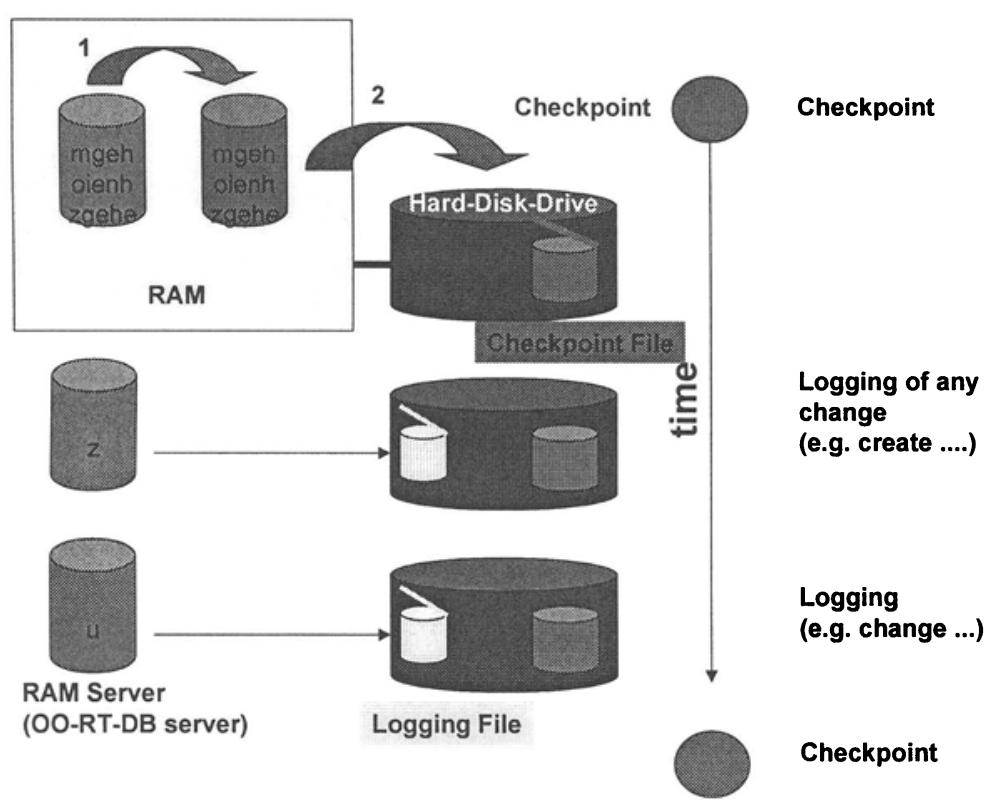

Figure 5 - Checkpoints and Logging Mechanism

Due to these measures, the risk that data could be lost - because it is held in the cache of the operating system and the cache of the HDD- is very minimal. The information which is available in these caches is not retrievable in the case that the power supply fails. With our servers this can only happen when a data package is processed by a server at exactly the same time as the power fails.

Power failures are much worse for a RDBMS because the files there are continuously open and so the probability that data get lost is much higher. Today some RDBMS use additional caches to accelerate the computing velocity. However the information available in these caches is not retrievable in the case that the power supply fails. As a result, the RDBMS can be corrupted when this happens.

\section{EXPERIENCES}

The distributed manufacturing system includes all functionality from getting the truck order to delivery involving dispatch notes, invoices etc. The capacity and production planning, the manufacturing execution for modules and variants in three locations, several warehouses in the production areas and lots of workplaces are realised with using the OO-RT-DB approach.

The system implementation time was extremely short ( 9 months) and the reliability is higher than $99,8 \%$. In Mannheim there are 8 server PC's running under UNIX which are mutually able to back-up the others and 70 workplaces using Windows PC's and 30 radio terminals directly connected to the servers.

The OO-RT-DB servers are also available under Windows NT and 2000 but for high reliability LINUX is preferred. 
The Internet connection of the OO-RT-DB is used for world-wide ordering spare parts. The customer subsidiary clicks on the needed modules of the truck shown in the web-browser and orders exactly what he wants. Through the Intranet the order is straight coming into the system and is automatically planned and produced. Up to now it took 4 weeks until the order has arrived passing different departments. From the former verbal description it was hard to see which part was really damaged.

\section{CONCLUSION}

The new Virtual Enterprise paradigm as well as Supply Chain Management and Distributed Manufacturing need novel approaches. There are many reports recently published which try to find general infrastructures for VE. For instance, the comprehensive work of (Camarinha-Matos, 1999 and Afsarmanesh, 1999) is based on RDBMS with an object-oriented information management system on top. By contrast the presented work is totally based on an object-oriented database which has proven extremely fast and reliable. All features such as privacy enforcement, information access rights, enterprise visibility levels, and transparency aspects are available and easy to realise because of the simple but very efficient structure of proposed OO-RT-DB. In our OO-system there is some data redundancy in contrast to theoretical theories to decouple systems and to make sites independent from others and the communication between both of them. Since we have proposed the OO-RTDB (Schaub, 1994, 1997, 1999) it was continuously improved and is not only 30 times faster than a RDBMS, needs $80 \%$ less resources, but also has proven to be very reasonable and the total cost of ownership is only half of that based on RDBMS systems. Therefore all customers who make use of the OO-RT-DB are totally convinced.

\section{Acknowledgements}

This work was funded in part by the European Commission, Esprit programme within the DELPHI project no. 26965. The authors also thank the valuable contributions from the consortium partners: DaimlerChrysler (Ger), Diconsult (Ger), Elite (I), ESPI (Ger), Sigikid (Ger), Tecnotron (P), Technical University Crete (Greece).

\section{REFERENCES}

1. Afsarmanesh, H., et al. -"Federated Information Management Requirements for Virtual Enterprises". In the PRO-VE'99 Book:"Infrastructures for Virtual Enterprises", Kluwer Academic Publishers, 1999

2. Camarinha-Matos, L.M., et al. - "Tendencies and General Requirements for Virtual Enterprises". In the PRO-VE'99 Book:"Infrastructures for Virtual Enterprises", Kluwer Academic Publishers, 1999

3. Schaub, G.-"Die Vorteile der objekt-orientierten Programmierung". - Magazine Die Fertigung, Volume 9, 1994

4. Schaub, G.-" Objekt-orientierte Software in der Fertigung". - Magazine Arbeitsvorbereitung, Munich, Volume 7, 1997

5. Schaub, G.,-“ An Internet Solution for Virtual Enterprises based on an Object-Oriented Real-Time Database".- In the BASYS 2000 Book:"Advances in Networked Enterprises", Kluwer Academic Publishers, 1999 